\title{
IgG4-related lymphadenopathy
}

Xiaobin Huang ${ }^{1}$, Wei Gao ${ }^{2}$, Jing Yang ${ }^{3}$, Xiaoling Yu ${ }^{4}$

${ }^{1}$ Department of General Medical, The First Affiliated Hospital of Jinzhou Medical University, Jinzhou, China

2Department of Rheumatology, The First Affiliated Hospital of Jinzhou Medical University, Jinzhou, China

${ }^{3}$ Department of Pathology, The First Affiliated Hospital of Jinzhou Medical University, Jinzhou, China

${ }^{4}$ Department of Geriatrics, The First Affiliated Hospital of Jinzhou Medical University, Jinzhou, China

Submitted: 30 January 2018

Accepted: 11 March 2018

Arch Med Sci Civil Dis 2018; 3: e21-e25

DOI: https://doi.org/10.5114/amscd.2018.74832

Copyright @ 2018 Termedia \& Banach

Immunoglobulin G4-related disease (IgG4-RD) is a recently described entity that is characterised by elevated serum IgG4 level, tissue infiltration, or tumefaction by lgG4-positive plasma cells, and it involves any organ in the body, including lacrimal glands, salivary glands, thyroid, lungs, lymph nodes, central nervous system, pituitary body, aorta, liver, pancreas, gall bladder, retroperitoneum, bile ducts, kidney, and prostate. Moreover, it is effected by steroid therapy for many patients with IgG4-RD [1-4]. Lymphadenopathy is frequently observed and sometimes appears as the first symptom in patients with IgG4-RD. IgG4-related lymphadenopathy has at least five histological subtypes, including multicentric Castleman disease-like (type I), reactive follicular hyperplasia-like (type II), interfollicular expansion and immunoblastosis (type III), progressively transformed germinal centres (PTGC)-type (type IV), and inflammatory pseudotumour (IPT)-like (type V). The diagnosis of IgG4-related lymphadenopathy is complicated [5].

We report a case of IgG4-related lymphadenopathy with multiorgan involvement. The differential diagnosis of IgG4-related lymphadenopathy is important to avoid misdiagnosis and mistreatment.

A 53-year-old female was admitted to our hospital because of multiple lymph nodes (neck, ear, supraclavicular, right axillary, inguinal) enlargement for 18 years, and swollen left eye for 2 months. Blood tests revealed low serum C3 and C4 levels and immunoglobulin G (IgG: $5064 \mathrm{mg} / \mathrm{dl})$, serum lgG4 (4520 mg/dl), anti-nuclear antibody (ANA, anti-dsDNA, immunofixation, HIV antibody, and Tumour markers were negative). Serum IgA, IgM, lactate dehydrogenase (LDH), and C-reactive protein (CRP) levels were all within normal limits. Urine tests showed no albuminuria or haematuria. However, the erythrocyte sedimentation rate (ESR) was higher than normal (Table I). Thoracic computed tomography (CT) showed mediastinal and right axillary lymph node enlargement with a small amount of pericardial effusion (Figures $1 \mathrm{~A}, \mathrm{~B}$ ).

Orbital CT revealed thickening of soft tissue around the orbit, left inferior rectus muscles, external rectus muscle, and subcutaneous of the eye rectus (Figure $1 \mathrm{C}$ ). Renal ultrasound showed double renal medulla thickening with multiple abnormal echoes, bilateral renal pelvis, and upper ureteral wall thickening with ureteropelvic migration. Lymph node ultrasound indicated bilateral submandibular, subauricular, axillary, and intra-

\author{
Corresponding author: \\ Xiaoling Yu \\ Department of Geriatrics \\ The First Affiliated \\ Hospital of Jinzhou \\ Medical University \\ No. 2, 5 Section of \\ People's Street \\ Jinzhou 121000, Liaoning \\ Province, China \\ Phone: +860416 4197632 \\ E-mail: xlyu8864@163.com
}


Table I. Main laboratory work-up, imaging, and histology studies from 1999 to 2017

\begin{tabular}{|c|c|c|}
\hline Year & Investigations & Results \\
\hline 1999 & Histology studies & Lymph node biopsy - reactive lymphoid hyperplasia \\
\hline \multirow[t]{3}{*}{2015} & Laboratory work-up & $\begin{array}{l}\text { Blood tests - blood routine, the function of the liver and kidney were normal, } \\
\text { HIV antibody and tumour markers were negative, low serum C3 and C4 levels, } \\
\qquad \operatorname{lgG}(47.8 \mathrm{~g} / \mathrm{l}) \text {, CRP }(0.65 \mathrm{mg} / \mathrm{l})\end{array}$ \\
\hline & Imaging studies & $\begin{array}{l}\text { Kidney ultrasound - a double renal diffuse lesion, a solid mass in the posterior } \\
\text { lower pole of the kidney, and a large possibility of enlargement of lymph nodes } \\
\text { in the posterior peritoneum } \\
\text { Abdominal ultrasound normal } \\
\text { Thoracic CT enlargement of the mediastinum and right axillary lymph node }\end{array}$ \\
\hline & Histology studies & $\begin{array}{l}\text { Bone marrow biopsy - suspicion of chronic lymphocytic proliferative disease } \\
\text { Lymph node biopsy - reactive lymphoid hyperplasia }\end{array}$ \\
\hline \multirow[t]{3}{*}{2017} & Laboratory work-up & $\begin{array}{c}\text { Blood tests: IgG (5064 mg/dl), serum lgG4 (4520 mg/dl), low serum C3 and C4 } \\
\text { levels, LDH (116 U/l), CRP (0.43 mg/l), ESR (113.00 mm/h), ANA, anti-dsDNA, } \\
\text { immunofixation, HIV antibody and tumour markers were negative } \\
\text { Urine tests - normal }\end{array}$ \\
\hline & Imaging studies & $\begin{array}{l}\text { Kidney ultrasound - double renal medulla thickening with multiple abnormal } \\
\text { echoes, bilateral renal pelvis and upper ureteral wall thickening with } \\
\text { ureteropelvic migration } \\
\text { Abdominal ultrasound suspicious of gallbladder wall thickening } \\
\text { Lymph node ultrasound - Bilateral submandibular, subauricular, axillary and } \\
\text { intraperitoneal lymphadenopathy. Diffuse lesions of the bilateral submandibular } \\
\text { glands and parotid glands } \\
\text { Thoracic CT - enlargement of the mediastinum and right axillary lymph nodes } \\
\text { with a small amount of pericardial effusion }\end{array}$ \\
\hline & Histology studies & Lymph node biopsy - suspicion of IgG4-related lymphadenopathy \\
\hline
\end{tabular}

HIV - human Immunodeficiency virus, IgG - immunoglobulin G, CRP - C-reactive protein, CT - computed tomography, $L D H$ - lactate dehydrogenase, ESR - erythrocyte sedimentation rate, anti-dsDNA - anti-double chain deoxyribonucleic acid antibodies, ANA - antinuclear antibodies.

peritoneal lymphadenopathy, and diffuse lesions of the bilateral submandibular glands and parotid glands. And abdominal ultrasound prompted suspicion of gallbladder wall thickening. Lymph node biopsy revealed characteristics of IgG4-related lymphadenopathy (Figures 2 A-D).

In 2015, the patient was admitted to the Department of Haematology in our hospital for multiple lymphadenopathy. Blood tests revealed that blood routine, CRP, and the function of the liver and kidney were normal. HIV antibody and tumour markers were negative. Serum C 3 and C4 levels were low, IgG was $4780 \mathrm{mg} / \mathrm{dl}$. Thoracic CT showed mediastinal and right axillary lymph node enlargement. Renal ultrasound suggested a double renal diffuse lesion, a solid mass in the posterior lower pole of the kidney, and a high probability of enlargement of lymph nodes in the posterior peritoneum. Lymph node biopsy revealed reactive lymphoid hyperplasia. Bone marrow biopsy indicated suspicion of chronic lymphocytic proliferative disease (Table I). At that time, the lymph nodes were slightly smaller after receiving four cycles of the CHOP regimen (cyclophosphamide $1.0 \mathrm{~g}$ first day, adriamycin $2 \mathrm{mg}$ first day, vincristine $60 \mathrm{mg}$ first day, and prednisone $60 \mathrm{mg} /$ day for 5 consecutive days each cycle).

In Addition, 18 years ago this patient had undergone lymphadenectomy for enlargement of multi- ple lymph nodes, and the postoperative pathology suggested reactive hyperplasia of lymph nodes (Table I). There had still been a swelling of the lymph nodes in different parts of the body since then.

We considered that the patient had multiple lymphadenopathy all over the body, and the emergence of submandibular gland, parotid gland, eye, kidney, and gallbladder multiple organ involvement. Serum IgG increased and serum IgG4 levels were significantly increased, and lymph node biopsy showed features of IgG4-related lymphadenopathy. Glucocorticoid (GC) combined cyclophosphamide (CYC) therapy (initial dose of prednisolone $50 \mathrm{mg} /$ day, and dose gradually reduced 1 month later; CYC $1.0 \mathrm{~g}$ once a month) and anti-osteoporosis therapy protected the liver and stomach treatment.

After 5 weeks, the patient's swollen lymph nodes were obviously smaller, and ESR and CRP were within the normal range. Serum C3, C4, IgG, and serum IgG4 levels were normal 4 months later. Thoracic CT showed that the right axillary lymph node and mediastinal lymph node were significantly smaller (Figures 1 D, E). Orbital CT suggested a slight reduction in the density around the eye (Figure $1 \mathrm{~F}$ ). Renal and abdominal ultrasound were normal. We considered the efficacy of GC combined with CYC, and it was still in the follow-up. 

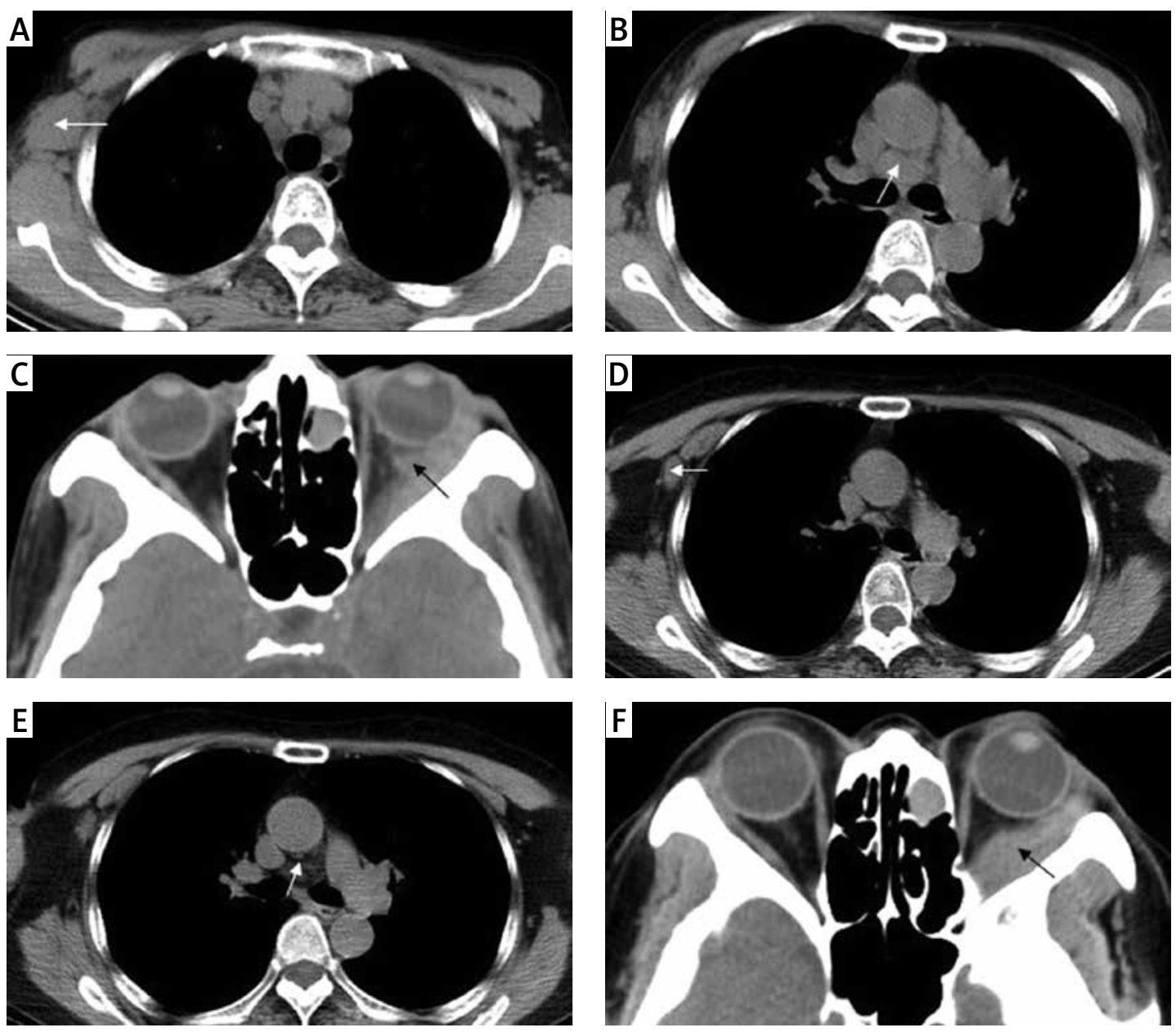

Figure 1. Thoracic CT showing a swollen right axillary lymph node (A; white arrow) and mediastinal lymph node (B; white arrow) whose sizes were approximately $28.02 \mathrm{~mm} \times 20.29 \mathrm{~mm}$ and $19.78 \mathrm{~mm} \times 12.20 \mathrm{~mm}$. Orbital CT revealed thickening of soft tissue around the orbit, left inferior rectus muscles, external rectus muscle, and subcutaneous rectus of the eye (C; black arrow). After treatment-thoracic CT revealed that the right axillary lymph nodes (D; white arrow) and the mediastinal lymph nodes (E; white arrow) were significantly smaller, whose sizes were approximately $16.34 \mathrm{~mm} \times 8.68 \mathrm{~mm}$ and $9.56 \mathrm{~mm} \times 4.32 \mathrm{~mm}$. Orbital CT suggested a slight reduction in the density around the eye ( $\mathrm{F}$; black arrow)

IgG4-related disease (IgG4-RD) is a rare fibro-inflammatory disorder that is characterised by pseudotumour-like lesions involving single or multiple organs. Different organs might be affected at the same time or one after another. Retroperitoneum, orbital pseudotumour, pancreas, salivary and lachrymal glands, and maxillary sinuses are the most frequently involved organs $[2,6]$.

The epidemiology of IgG4-RD is rarely reported. Uchida et al. estimated that the incidence of IgG4-RD throughout Japan is $0.28-1.08 / 100,000$. In these patients, the male : female ratio is $1: 0.77$. The median age of disease onset is 58.8 years, and there are 336-1300 patients newly diagnosed per year [7].

The diagnostic criteria of IgG4-RD are as follows: (1) organ enlargement, mass or nodular lesions, or organ dysfunction; (2) elevated serum lgG4 levels (> $135 \mathrm{mg} / \mathrm{dl}$ ); and (3) histopathological findings of lgG $4+/ \operatorname{lgG}+$ cell ratio $>40 \%$ and at least > 10 lgG4 cells/high-powered field (HPF). The patients meeting all the criteria were "definite" (1), (2), and (3), if criteria (1) and (2) is "possible", and "probable" in patients with criteria (1) and (3) satisfied [8]. According to these criteria, our case was more likely to be "possible". However, lymph node biopsy suggested multicentric Castleman disease-like features, and the patient was sensitive to glucocorticoid combined with cyclophosphamide. Perhaps experimental therapy can be used as an auxiliary diagnostic criterion when the diagnosis of IgG4-RD is difficult.

The differential diagnosis of lymphadenopathy is extremely important, including lymphoma, multicentric Castleman disease (MCD), metastatic malignant tumour and other immune-mediated or haematological diseases. It is particularly similar to the pathological features of plasma cell-type Castleman disease (CD). A large number of IgG4-positive plasma cells, infiltration of eosinophils among 

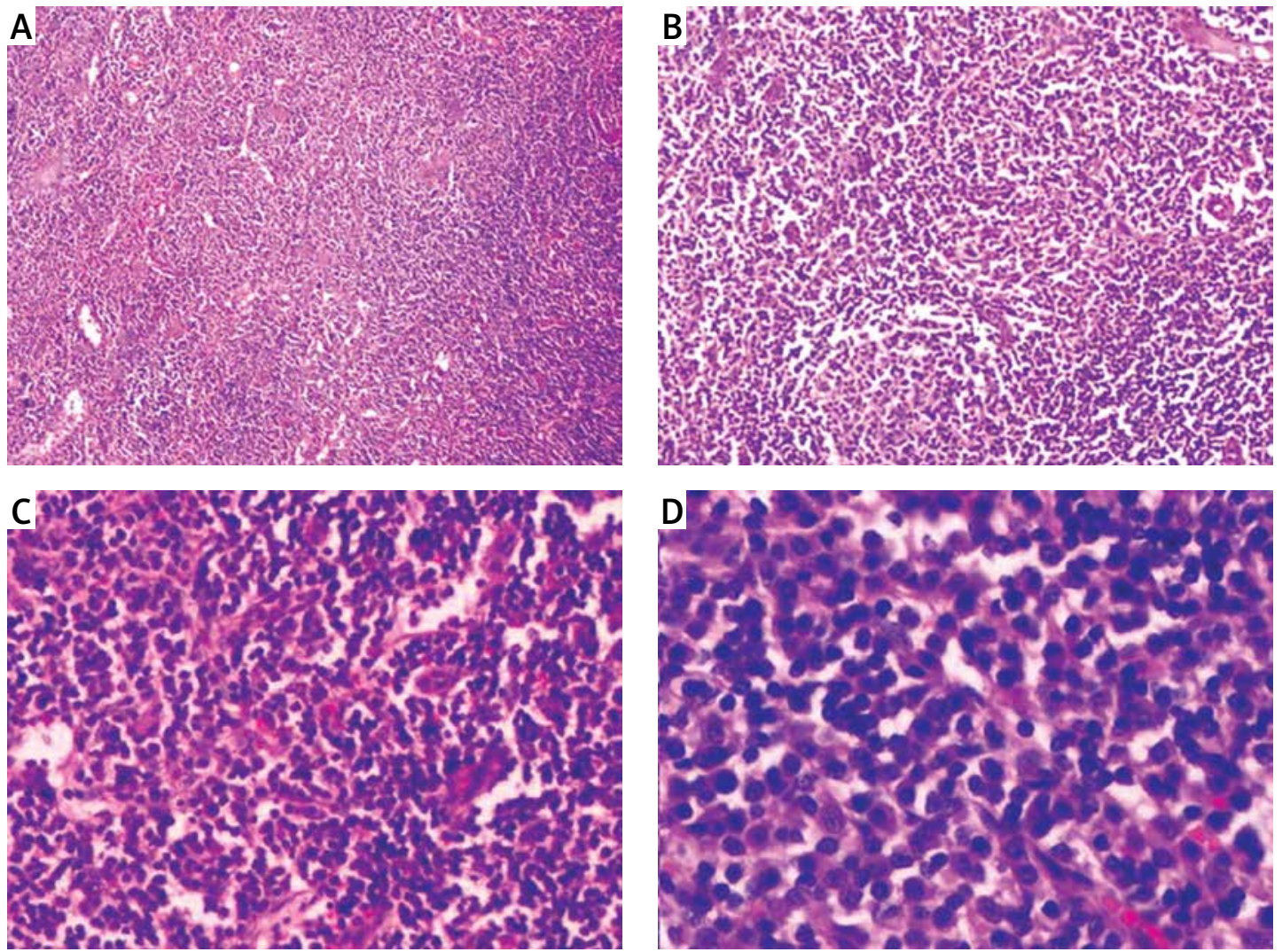

Figure 2. Lymph node biopsy revealed histological subtype of IgG4-related lymphadenopathy (multicentric Castleman disease-like) (A-40x; B - 100x; C - 200x; D -400x). Different sizes lymphoid follicular structures, the area of the follicles was obvious. Some areas of the follicles were enlarged, and the lymphatic sinuses were open. There were numerous small lymphocytes, plasma cells, and activated immunocytes

follicles, not associated with fever and weight loss, and aging. Serum lactate dehydrogenase, interleukin-6 (IL-6), and CRP levels were normal or only slightly elevated. Other laboratory findings included elevated serum IgG4, serum IgG, and ESR levels. More support IgG4-related lymphadenopathy when these characteristics are found $[5,9,10]$. Furthermore, MCD could also have elevated serum IgG4 levels. But laboratory findings of anaemia, hypoalbuminaemia, polyclonal gammaglobulinaemia, high CRP level, and elevated IL-6 level are all consistent with the MCD [11].

Although there was no immunohistochemistry in our case, according to multiple lymph node enlargement and multiple organ involvement in the whole body. Serum IgG serum IgG4 levels were significantly increased. Lymph node biopsy showed features of IgG4-related lymphadenopathy and identified with other diseases. What is more, the case was effective with glucocorticoid (GC) combined CYC. Therefore, we diagnosed IgG4-related lymphadenopathy. It is worth noting that scholars should pay more attention to the study of the aetiology and diagnostics of IgG4$\mathrm{RD}$ in future research, to avoid misdiagnosis and misdiagnosis and to improve the detection rate of patients.
Currently, there are no randomised controlled trials to evaluate treatment options. GC are regarded as the first-line therapy, and the majority of IgG4-RD patients respond favourably [1, 12]. In general, most experts suggest a starting dose for prednisolone taken orally of $40 \mathrm{mg}$ or $0.6 \mathrm{mg} / \mathrm{kg}$ of body weight per day for 2-4 weeks. GCs should be reduced slowly after induction of treatment and last for 3-6 months [13, 14]. However, some experts recommend long-term maintenance therapy at a dose between 2.5 and $5 \mathrm{mg} /$ day for up to 3 years [15].

Fei et al. indicated that GC combined CYC treatment had better effect and lower relapse rate than GC alone for IgG4-RD patients. Moreover, if patients have more than six organs affected, GC and immunosuppressant agent treatment at the onset should be chosen [16]. Our case showed good response to GC combined with CYC. A French multicentre study suggested that rituximab (RTX) is an effective treatment for both remission induction and treatment of relapses in IgG4-RD. Systematic RTX retreatment could represent a therapeutic strategy to prevent relapse. However, considering the occurrence of hypogammaglobulinaemia and the high rate of severe infections in some patients [17], more cases need to be accumulated to clarify these issues. 
In conclusion, we encountered a case with IgG4-related lymphadenopathy with multi-system involvement, and treatment using GC combined with CYC had a good effect. Few cases of IgG4-related lymphadenopathy with multiorgan involvement, as in this case, have been reported. Long-term follow-up is indispensable to monitor additional organ involvement and relapses.

\section{Conflict of interest}

The authors declare no conflict of interest.

\section{References}

1. Pieringer H, Parzer I, Wohrer A, Reis P, Oppl B, Zwerina J. IgG4-related disease: an orphan disease with many faces. Orphanet J Rare Dis 2014; 9: 110.

2. Fernandez-Codina A, Martinez-Valle F, Pinilla B, et al. IgG4-related disease: results from a Multicenter Spanish Registry. Medicine 2015; 94: e1275.

3. Stone JH, Zen Y, Deshpande V. IgG4-related disease. N Engl J Med 2012; 366: 539-51.

4. Umehara H, Okazaki K, Masaki Y, et al. A novel clinical entity, IgG4-related disease (IgG4RD): general concept and details. Mod Rheumatol 2012; 22: 1-14.

5. Sato Y, Yoshino T. IgG4-Related Lymphadenopathy. Int J Rheumatol 2012; 2012: 572539.

6. Okazaki K, Umehara H. Are classification criteria for IgG4-RD now possible? The concept of IgG4-related disease and proposal of comprehensive diagnostic criteria in Japan. Int J Rheumatol 2012; 2012: 357071.

7. Uchida K, Masamune A, Shimosegawa T, Okazaki K. Prevalence of IgG4-related disease in Japan based on Nationwide Survey in 2009. Int J Rheumatol 2012; 2012: 358371.

8. Umehara H, Okazaki K, Masaki Y, et al. Comprehensive diagnostic criteriafor lgG4-related disease (IgG4-RD), 2011. Modern Rheumatology 2012; 22: 21-30.

9. Cheuk W, Yuen HK, Chu SY, Chiu EK, Lam LK, Chan JK. Lymphadenopathy of IgG4-related sclerosing disease. Am J Surg Pathol 2008; 32: 671-81.

10. Sato Y, Kojima M, Takata K, et al. Systemic IgG4-related lymphadenopathy: a clinical and pathologic comparison to multicentric Castleman disease. Mod Pathol 2009; 22: 589-99.

11. Takeuchi M, Sato Y, Takata K, et al. Cutaneous multicentric Castleman disease mimicking lgG4-related disease. Pathol Res Pract 2012; 208: 746-9.

12. Brito-Zeron P, Kostov B, Bosch X, Acar-Denizli N, RamosCasals M, Stone JH. Therapeutic approach to IgG4-related disease. Medicine 2016; 95: e4002.

13. Khosroshahi A, Wallace ZS, Crowe JL, et al. International consensus guidance statement on the management and treatment of IgG4-related disease. Arthritis Rheumatol 2015; 67: 1688-99.

14. Dhobale S, Bedetti C, Killian P, et al. IgG4 related sclerosing disease withmultiple organ involvements and response to corticosteroid treatment. J Clin Rheumatol 2009; 15: 354-7.

15. Kamisawa T, Okazaki K, Kawa S, et al. Amendment of the Japanese consensus guidelines for autoimmune pancreatitis, 2013 III. Treatment and prognosisof autoimmune pancreatitis. J Gastroenterol 2014; 49: 961-70.

16. Yunyun F, Yu C, Panpan Z, et al. Efficacy of cyclophosphamide treatment for immunoglobulin G4-related disease with addition of glucocorticoids. Sci Rep 2017; 7: 6195.
17. Ebbo M, Grados A, Samson M, et al. Long-term efficacy and safety of rituximab in IgG4-related disease: data from a French nationwide study of thirty-three patients. PLoS One 2017; 12: e0183844. 